
U.Heber ${ }^{1}$, K. Hunger ${ }^{1}$, R.P. Kudritzki ${ }^{2}$, K. P. Simon ${ }^{2}$
1) Institut für Theor. Physik und Sternwarte, Kiel
2) Institut für Astronomie und Astrophysik, München

Among the faint blue halo stars the sdo's form a group whose evolutionary status is still not fully understood. Phenomenologically they appear to be related to the horizontal branch. The knowledge of stellar parameters and abundances may give a clue for understanding their evolutionary status. As the sdo's are hot ( $\mathrm{T}$ eff $\geq 35000 \mathrm{~K})$, the assumption of LTE leads to substantial errors. Hence detailed NLTE analyses are inevitable.

\title{
THE METHOD OF ANALYSIS
}

High (or medium) resolution visual and UV spectra are analysed by means of complete NLTE techniques in the following way:

In the first step a grid of NLTE model atmospheres is constructed which is then used for additional line formation calculations for hydrogen, He II and He I. A fit of the observed profiles of hydrogen and helium lines then yields simultaneously effective temperature, gravity and helium abundance. From the finally adopted model multi-level (or twolevel) calculations are carried out for different metal ions to determine metal abundances. An illustrative example for the procedure of model fitting is given by Kudritzki and Simon (1978).

\section{ATMOSPHERIC PARAMETERS}

26 sdo's up to now have been analyzed for atmospheric parameters. The results are summarized in Table 1 and plotted in Fig.1. Most strikingly there is a subdivision of the group with respect to the helium content. We find three groups:

1) 5 stars are extremely helium rich. Their photospheres consist almost entirely of helium (helium number fraction $y=1.0$ ).

2) 10 stars have intermediate helium rich photospheres $(y \approx 0.5)$.

3) 11 stars are helium poor $(0.0007 \leqslant y \leq 0.12)$.

Moreover, there is a clear borderline which separates helium rich objects from the helium poor ones, the critical temperature being $40000 \mathrm{~K}$. The helium rich sdo's are hotter, the helium poor ones cooler then this. The helium poor sdo's are intermediate in $\mathrm{T}$ eff to the sdB stars (see Fig.1) which are also helium poor and are better called sdoB stars. 
Table 1:Atmospheric parameters of sdo stars (for a list of references see Heber et al., 1983b)

\begin{tabular}{|c|c|c|c|c|c|c|c|}
\hline star & $\mathrm{T}_{\text {eff }} / \mathrm{K}$ & $\log g$ & $y$ & star & $\mathrm{T}_{\text {eff }} / \mathrm{K}$ & $\log g$ & Y \\
\hline$C D-31^{\circ} 4800$ & 42500 & 5.5 & 1.0 & $\mathrm{BD}-3^{\circ} 2179$ & $>55000$ & 4.5 & 0.3 \\
\hline $\mathrm{BD}+39^{\circ} 3226$ & 45000 & 5.5 & 1.0 & 1566 & 42600 & 5.0 & 0.2 \\
\hline $\mathrm{BD}+37^{\circ} \quad 442$ & 55000 & 4.0 & 1.0 & 884 & 40000 & 6.1 & 0.12 \\
\hline TONS & $40000:$ & $6.5:$ & 1.0 & 169 & 37000 & 6.3 & 0.04 \\
\hline SB & 36300 & 5.4 & 1.0 & HD 149382 & 35000 & 5.5 & 0.04 \\
\hline 58 & 38000 & 4.5 & 0.7 & Feige 110 & 40000 & 5.0 & 0.03 \\
\hline HD 127493 & 42500 & 5.25 & 0.6 & $\mathrm{SB}$ & 39000 & 5.4 & 0.03 \\
\hline $\mathrm{BD}+75^{\circ} \quad 325$ & 55000 & 5.3 & 0.6 & EG & 38000 & 5.8 & 0.02 \\
\hline 49798 & 47500 & 4.25 & 0.5 & Feige 66 & 36000 & 6.0 & 0.02 \\
\hline 705 & 44700 & 5.8 & 0.5 & SN 1006 & $38500:$ & $6.5:$ & 0.015 \\
\hline 630 & 55000 & 6.0 & 0.5 & 3241 & 41000 & 5.7 & $\leq 0.005$ \\
\hline 933 & 49000 & 5.5 & 0.4 & 3459 & 40000 & 5.3 & 0.003 \\
\hline 128220 & $\{45000$ & 4.75 & 0.37 & 707 & 34000 & 6.0 & $\leq 0.000^{\circ}$ \\
\hline
\end{tabular}

METAL ABUNDANCES

Abundances of $\mathrm{C}, \mathrm{N}$ and $\mathrm{Si}$ have been obtained for 3 helium rich sdo's and 3 sdOB's. The results are listed in Table 2. For the helium rich sdo's the data given in Table 2 indicate that the material in their atmospheres has been processed in the CNO cycle. For the sdoB's the abundances are considerably different (see Table 2). Silicon is deficient in all analyzed stars by large factors. Nitrogen is approximately solar. Carbon is moderately deficient in HD 149382 and Feige 66 while it is strongly deficient in Feige 110 (by more than a factor of 300000). These strange abundance patterns of the sdoB stars can be explained by diffusion. Due to the high gravity helium, carbon and silicon settle downwards out of the photosphere. The prerequisite for gravitational settling is an atmosphere that is quiet. For effective temperatures near $40000 \mathrm{~K}$ a helium convection zone occurs, which may impede diffusion.

Table 2: Abundances of sdo stars (log of mass fraction)

\begin{tabular}{|c|c|c|c|c|c|c|}
\hline star & $\mathrm{H}$ & $\mathrm{He}$ & $\mathrm{C}$ & $\mathrm{N}$ & $\mathrm{Si}$ & \\
\hline 49798 & -0.70 & -0.10 & -3.7 & -1.6 & -3.0 & \\
\hline HD 127493 & -0.85 & -0.07 & -4.1 & -2.1 & -3.0 & \\
\hline $\mathrm{BD}+750325$ & -0.85 & -0.07 & -3.7 & -1.9 & -3.1 & \\
\hline CNO cycle & -0.85 & -0.07 & $-4 \cdot 1$ & -1.9 & & (with $\mathrm{T}_{6}=2 \cdot 10^{7} \mathrm{~K}$ ) \\
\hline sdoB stars: & & & & & & \\
\hline HD 149382 & -0.07 & -0.85 & -4.4 & -2.9 & $<-7.7$ & \\
\hline Feige & -0.03 & -1.12 & $-4 \cdot 5$ & -2.7 & $<-7.8$ & \\
\hline $\begin{array}{l}\text { Feige } 100 \\
\text { sun }\end{array}$ & $\begin{array}{l}-0.05 \\
-0.152\end{array}$ & $\begin{array}{l}-0.96 \\
-0.55\end{array}$ & $\begin{array}{l}<-7.9 \\
-2.4\end{array}$ & $\begin{array}{l}-3.1 \\
-3.0\end{array}$ & $\begin{aligned}< & -6.8 \\
& -3.16\end{aligned}$ & \\
\hline
\end{tabular}




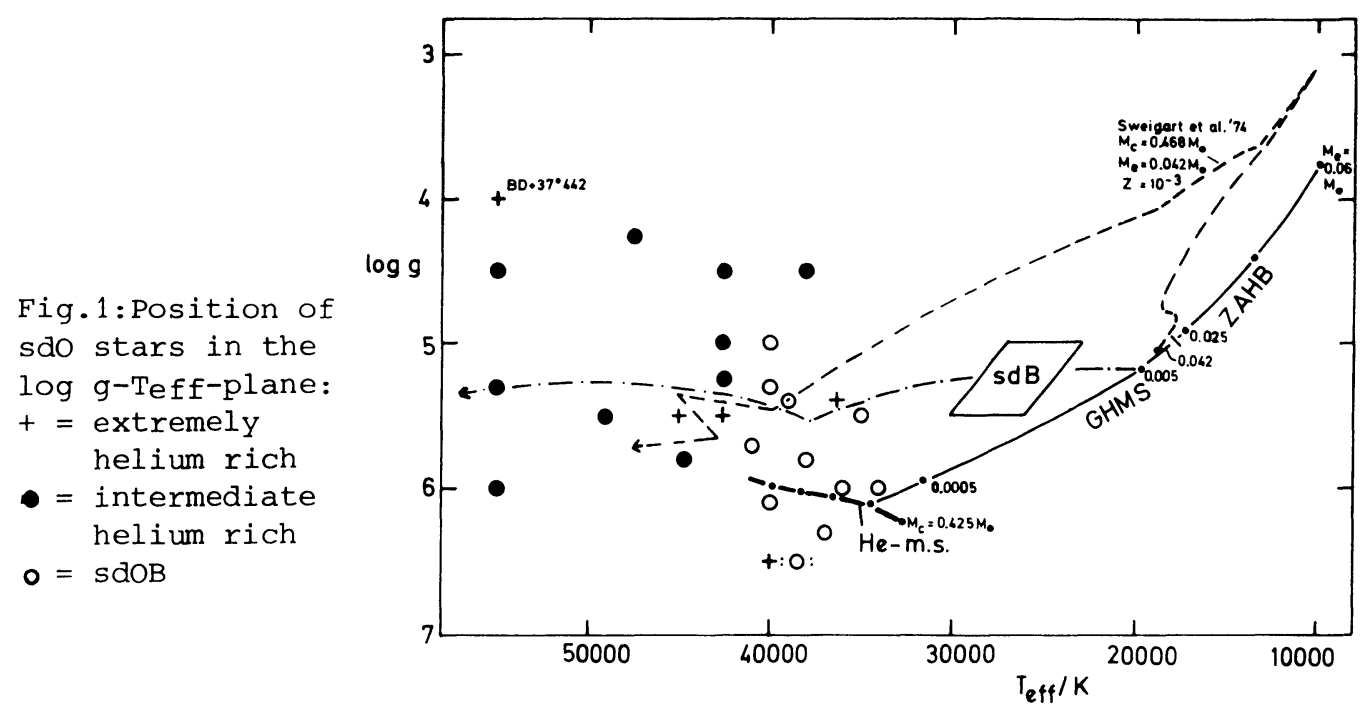

THE EVOLUTIONARÝ STATUS OF THE SDO STARS

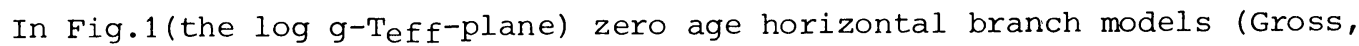
1973) are plotted.The models are labelled with their envelope masses $\mathrm{M}_{\mathrm{e}}$. For $\mathrm{M}_{e}<0.02 \mathrm{M}_{\odot}$ the hydrogen rich envelope is inert. These models may be called generalized helium main sequence models (GHMS, see Heber et al., 1983a). The true helium main sequence is also shown. The sdo's lie to the left of the ZAHB and GHMS but above the helium main sequence. We can reach the position of the subdwarfs in the log-Teff-plane when evolution is considered. Two relevant evolutionary tracks are shown in Fig.1. The dashed curve represents evolution of a ZAHB-star (Sweigart et al., 1974), while a GHMS star probbly evolves similar to a pure helium star along the dashed dotted curve. From Fig.1 we conclude that there are essentially two ways sdo's can form: either by evolution from horizontal branch B stars and/or from sdB stars. The latter are slightly evolved GHMS stars $\left(\mathrm{M}_{\mathrm{e}}<0.02 \mathrm{M}_{\odot}\right)$. Some of the high gravity sdOB's are located at the helium main sequence, which means that their hydrogen rich envelope masses are even smaller $\left(\mathrm{M}_{\mathrm{e}}<10^{-3} \mathrm{M}_{\odot}\right)$. This situation is reminiscent of DA white dwarfs which are known to have very little hydrogen floating atop the helium rich layers. Some (low gravity) stars (e.g. $\mathrm{BD}+370442)$ are probably remnants of the asymptotic giant branch.

\section{REFERENCES :}

Gross,P.G.: 1973, Monthly Notices Roy. Astron. Soc. 164, 65 Heber,U., Hunger,K., Jonas,G., Kudritzki,R.P.: 1983a, Astron. Astrophys., in press

Heber,U., Hamann,W.-R., Hunger,K., Kudritzki,R.P., Simon,K.P. Mendez,R.H.: 1983b, Astron. Astrophys., submitted

Kudritzki,R.P., Simon, K.P.: 1978, Astron. Astrophys. 70, 653

Sweigart,A.V., Mengel,J.G., Demarque,P.: 1974, Astron. Astrophys. 30, 13 


\section{DISCUSSION}

Renzini: How can an $\mathrm{HB}$ star be turned into an extreme He star?

Heber: No idea.

Cox: What are the luminosities above which there is convection to prevent the He settling?

Heber: The onset of convection in the photosphere is not very sensitive to luminosity. Instead it is more sensitive to the effective temperature of the star.

Mould: Isn't it possible that an extremely large mass loss could have completely stripped the hydrogen envelope from these stars?

Heber: Yes. In order to account for the very low envelope masses $M_{e}$ of the subdwarfs $\left(\mathrm{M}_{\mathrm{e}} \leqslant 0.05 \mathrm{M}_{\odot}\right)$ we have to consider mass loss both on the red giant branch and during the core helium flash. At present nobody is able to tell you what is going on during the core helium flash.

Schatzman: Is there any information on the rotational velocities of these objects?

Heber: From our spectra we conclude that they must be very low (below the detection limit). 